TAIWANESE JOURNAL OF MATHEMATICS

Vol. 17, No. 4, pp. 1245-1266, August 2013

DOI: $10.11650 /$ tjm.17.2013.2617

This paper is available online at http://journal.taiwanmathsoc.org.tw

\title{
HÖLDER CONTINUITY OF THE SOLUTION MAP TO AN ELLIPTIC OPTIMAL CONTROL PROBLEM WITH MIXED CONSTRAINTS
}

\author{
V. H. Nhu, N. H. Anh and B. T. Kien
}

\begin{abstract}
The goal of the paper is to investigate the Hölder continuity of the solution map to a parametric optimal control problem which is governed by elliptic equations with mixed control-state constraints and convex cost functions. By reducing the problem to a programming problem and parametric variational inequality, we get sufficient conditions under which the solution map is Hölder continuous in parameters.
\end{abstract}

\section{INTRODUCTION}

Let $\Omega$ be a bounded domain in $R^{N}$ with the Lipschitz boundary $\partial \Omega$ and $N \in\{2,3\}$. We consider the following parametric optimal control problem for the elliptic equations with mixed control-state constraints:

Find a control function $u \in L^{p}(\Omega), p \geq 2$ and a state $y \in H_{0}^{1}(\Omega) \cap C(\bar{\Omega})$ which minimize the cost

$$
F(y, u, \mu)=\int_{\Omega} f(x, y(x), u(x), \mu(x)) d x
$$

with the state equation

$$
\begin{cases}A y=u+\lambda_{1} & \text { in } \Omega \\ y=0 & \text { on } \partial \Omega\end{cases}
$$

and pointwise constraints

$$
\begin{cases}u \geq \lambda_{2} & \text { in } \Omega \\ \epsilon u \geq \delta y+\lambda_{3} & \text { in } \Omega,\end{cases}
$$

Received November 13, 2012, accepted January 18, 2013.

Communicated by Neil Trudinger.

2010 Mathematics Subject Classification:

Key words and phrases: Parametric optimal control, Hölder continuity, Variational inequality, Elliptic equation. 
where $f: \Omega \times R \times R \times R^{k} \rightarrow R \cup\{+\infty\}$ with $k \geq 1$, is given function,

$$
\mu=\left(\mu_{1}, \ldots, \mu_{k}\right) \in L^{\infty}(\Omega)^{k}, \lambda=\left(\lambda_{1}, \lambda_{2}, \lambda_{3}\right) \in L^{p}(\Omega) \times L^{\infty}(\Omega) \times L^{\infty}(\Omega)
$$

are parameters, $A$ denotes a second-order elliptic operator of the form

$$
A y(x)=-\sum_{i, j=1}^{N} D_{j}\left(a_{i j}(x) D_{i} y(x)\right)+a_{0}(x) y(x),
$$

where coefficients $a_{i j} \in L^{\infty}(\Omega)$ satisfy the strongly elliptic condition

$$
\sum_{i, j=1}^{N} a_{i j}(x) \xi_{i} \xi_{j} \geq \lambda_{A}|\xi|^{2} \quad \forall \xi \in R^{N}, \text { a. e. } x \in \Omega
$$

for some $\lambda_{A}>0$ and $a_{0} \in L^{\infty}(\Omega), a_{0}(x) \geq 0$ almost everywhere $x \in \Omega, \delta \in L^{\infty}(\Omega)$ and $\epsilon \in L^{\infty}(\Omega)$.

Let us put

$$
Y=H_{0}^{1}(\Omega) \cap C(\bar{\Omega}), U=L^{p}(\Omega), Z=Y \times U
$$

and

$$
M=L^{\infty}(\Omega)^{k}, \Lambda=L^{p}(\Omega) \times L^{\infty}(\Omega) \times L^{\infty}(\Omega) .
$$

The norms of $y \in Y, \mu \in M$ and $\lambda \in \Lambda$ are defined by

$$
\begin{aligned}
\|y\|_{Y} & =\|y\|_{H_{0}^{1}(\Omega)}+\|y\|_{C(\bar{\Omega})}, \quad\|\mu\|_{M}=\max \left\{\left\|\mu_{i}\right\|_{L^{\infty}(\Omega)}: 1 \leq i \leq k\right\} \\
\text { and } \quad\|\lambda\|_{\Lambda} & =\left\|\lambda_{1}\right\|_{L^{p}(\Omega)}+\left\|\lambda_{2}\right\|_{L^{\infty}(\Omega)}+\left\|\lambda_{3}\right\|_{L^{\infty}(\Omega)},
\end{aligned}
$$

respectively.

In the sequel, we denote by $B_{X}$ and $\bar{B}_{X}$ the open unit ball and the closed unit ball in a norm space $X$, respectively. Also, given $x \in X$ and $\delta>0, B_{X}(x, \delta)$ and $\bar{B}_{X}(x, \delta)$ stand for an open ball and a closed ball, respectively with center $x$ and radius $\delta$.

Let us define a set-valued map $K: \Lambda \rightrightarrows Z$ by setting

$$
K(\lambda)=\{z=(y, u) \in Y \times U \mid(2) \text { and (3) are satisfied }\} .
$$

Then problem (1)-(3) can be formulated in the form

$$
P(\mu, \lambda) \quad\left\{\begin{array}{l}
F(z, \mu) \rightarrow \inf \\
z \in K(\lambda) .
\end{array}\right.
$$

We denote by $\mathcal{S}(\mu, \lambda)$ the solution set of $P(\mu, \lambda)$. In this paper, we always assume that $\mathcal{S}(\bar{\mu}, \bar{\lambda})=\{\bar{z}\}$, that is, problem $P(\bar{\mu}, \bar{\lambda})$ has a unique solution $\bar{z}=\bar{z}(\bar{\mu}, \bar{\lambda})=$ $(\bar{y}(\bar{\mu}, \bar{\lambda}), \bar{u}(\bar{\mu}, \bar{\lambda}))$. 
Our main concern is to investigate the behavior of $\mathcal{S}(\mu, \lambda)$ when $(\mu, \lambda)$ varies around $(\bar{\mu}, \bar{\lambda})$. This problem interested some authors in the last decade. For papers which have a closed connection to the present work, we refer the readers to $[2,10,15$, $16]$ and the references given therein. When $f$ is a quadratic function, that is

$$
f(x, y, u, \mu)=\frac{1}{2}\left|y-y_{d}(x)\right|^{2}+\frac{\gamma}{2}\left|u-u_{d}(x)\right|^{2}-\mu_{1} y-\mu_{2} u,
$$

where $y_{d}$ and $u_{d}$ are given in $L^{2}(\Omega)$, and $\gamma>0$ is a constant, [2,10] and [15] showed that the solution map is singleton and Lipschitz continuous in parameters.

It is noted that the obtained result of [10] is for problem with pure state constraints, the obtained result of [15] is for problem with pure control constraints while the obtained result of [2] is for problem with mixed control-state constraints (3) with $\epsilon=\epsilon_{0}, \delta=-1$ and under additional condition that

$$
\exists \sigma>0, \quad S_{1}^{\sigma} \cap S_{2}^{\sigma}=\emptyset,
$$

where

$$
\begin{gathered}
S_{1}^{\sigma}:=\left\{x \in \Omega: 0 \leq \bar{u}_{0}(x) \leq \sigma\right\}, \\
S_{2}^{\sigma}:=\left\{x \in \Omega: 0 \leq \epsilon_{0} \bar{u}_{0}(x)+\bar{y}_{0}(x)-y_{c}(x) \leq \sigma\right\}
\end{gathered}
$$

with $y_{c} \in L^{\infty}(\Omega),\left(\bar{y}_{0}, \bar{u}_{0}\right)$ is a solution of $P(\bar{\mu}, \bar{\lambda})$ corresponding to $\bar{\mu}=0$ and $\bar{\lambda}=\left(0,0, y_{c}\right)$.

In this paper we continue to develop results of [2] by considering problem (1)-(3) under weaker conditions and for a larger class of cost functions $F$, where the integrand function $f$ is not necessary to be quadratic. Namely, by reducing (1)-(3) to a parametric variational inequality and using technique in [8] and [18], we will show that, under certain conditions but without condition (6), the solution map $\mathcal{S}$ of problem (1)-(3) is singleton and Hölder continuous in $(\mu, \lambda)$.

Let us recall some concepts which are related to our problems. Given a function $\phi \in L^{2}(\Omega)$, a function $y \in H_{0}^{1}(\Omega)$ is called a weak solution of the elliptic partial differential equation

$$
\left\{\begin{array}{l}
A y=\phi \quad \text { in } \Omega \\
y=0 \text { on } \partial \Omega
\end{array}\right.
$$

if

$\int_{\Omega}\left(\sum_{i, j=1}^{N} a_{i j}(x) D_{i} y(x) D_{j} v(x)+a_{0}(x) y(x) v(x)\right) d x=\int_{\Omega} \phi(x) v(x) d x \quad \forall v \in H_{0}^{1}(\Omega)$.

Given a Banach space $E$ and a nonempty closed convex set $K$ in $E$, the normal cone to $K$ at a point $z_{0} \in Z$ is define by

$$
N\left(z_{0} ; K\right)=\left\{z^{*} \in E^{*}:\left\langle z^{*}, z-z_{0}\right\rangle \leq 0, \forall z \in K\right\} .
$$


For definition of normal cones and their properties, we refer the readers to [13, Chapter 4].

Let us impose the following conditions for problem (1)-(3).

( $A 1) \Omega$ is a bounded domain in $R^{N}, N \in\{2,3\}$, with the Lipschitz boundary $\partial \Omega$ and $\epsilon, \delta \in L^{\infty}(\Omega), \epsilon(x) \geq \epsilon_{0}>0$, a.e. $x$ in $\Omega$.

(A2) $f(\cdot, y, u, \mu)$ is measurable for all $(y, u, \mu) \in R \times R \times R^{k}$ and $f(x, \cdot, \cdot, \cdot)$ is continuous a.e. $x$ in $\Omega$. Besides, there exist a positive number $\epsilon_{1}$ and a continuous nonnegative function $g: \bar{\Omega} \times R^{3} \rightarrow R$ such that for all $(x, \mu) \in \Omega \times R^{k}$ with $|\mu-\bar{\mu}(x)| \leq \epsilon_{1}$, one has

$$
\begin{aligned}
\mid f(x, \bar{y}(x), \bar{u}(x), \mu)-f(x, & \bar{y}(x), \bar{u}(x), \bar{\mu}(x)) \mid \\
& \leq g(x,|\bar{y}(x)|,|\mu|,|\bar{\mu}(x)|) H_{1}(|\bar{u}(x)|),
\end{aligned}
$$

where $H_{1}(\cdot)$ is the following form

$$
H_{1}(t)=\sum_{i=1}^{m_{1}} t^{s_{i}} \quad \text { with } \quad m_{1} \geq 1,0 \leq s_{i} \leq p, \forall i=\overline{1, m_{1}} .
$$

(A3) There exist constant numbers $\epsilon_{2}, \rho>0$ such that for a. e. $x \in \Omega$ the function $(y, u) \mapsto f(x, y, u, \mu)$ is continuously differentiable and convex on subset $D(x)$ and the following condition holds

$$
\left(f_{z}\left(x, z_{1}, \mu\right)-f_{z}\left(x, z_{2}, \mu\right)\right)\left(z_{1}-z_{2}\right) \geq \rho\left|u_{1}-u_{2}\right|^{p}
$$

for all $z_{i}=\left(y_{i}, u_{i}\right) \in D(x)$ and for all $\mu \in R^{k}$ with $|\mu-\bar{\mu}(x)| \leq \epsilon_{1}$, where $D(x)=\left(\bar{y}(x)-\epsilon_{2}, \bar{y}(x)+\epsilon_{2}\right) \times R$.

(A4) There exist continuous functions $a_{i}: \bar{\Omega} \times R^{2} \rightarrow R, b_{i}: \bar{\Omega} \times R^{3} \rightarrow R$ and positive numbers $\alpha_{i}, i=1,2$ such that

$$
\begin{aligned}
\left|f_{y}(x, y, u, \bar{\mu}(x))\right| & \leq a_{1}(x,|y|,|\bar{\mu}(x)|) H_{1}(|u|), \\
\left|f_{u}(x, y, u, \bar{\mu}(x))\right| & \leq a_{2}(x,|y|,|\bar{\mu}(x)|) H_{2}(|u|)
\end{aligned}
$$

for all $x \in \Omega, y, u \in R$ satisfying $|y-\bar{y}(x)| \leq \epsilon_{2}$ and

$$
\begin{aligned}
\left|f_{y}\left(x, y, u, \mu^{1}\right)-f_{y}\left(x, y, u, \mu^{2}\right)\right| & \leq b_{1}\left(x,|y|,\left|\mu^{1}\right|,\left|\mu^{2}\right|\right) H_{1}(|u|)\left|\mu^{1}-\mu^{2}\right|^{\alpha_{1}}, \\
\left|f_{u}\left(x, y, u, \mu^{1}\right)-f_{u}\left(x, y, u, \mu^{2}\right)\right| & \leq b_{2}\left(x,|y|,\left|\mu^{1}\right|,\left|\mu^{2}\right|\right) H_{2}(|u|)\left|\mu^{1}-\mu^{2}\right|^{\alpha_{2}}
\end{aligned}
$$

for all $x \in \Omega, y, u \in R, \mu^{i} \in R^{k}$ satisfying $\left|\mu^{i}-\bar{\mu}(x)\right| \leq \epsilon_{1}, i=1,2,|y-\bar{y}(x)| \leq \epsilon_{2}$, where

$$
H_{2}(t)=\sum_{j=1}^{m_{2}} t^{s_{j}} \quad \text { with } m_{2} \geq 1,0 \leq s_{j} \leq p-1 \forall j=\overline{1, m_{2}}
$$


Under conditions $(A 1),(A 2)$ and by Lemma 2.1 , for each $\phi \in L^{p}(\Omega)$, equation (7) has a unique solution $y_{\phi} \in H_{0}^{1}(\Omega) \cap C(\bar{\Omega})$ which satisfies the estimation

$$
\left\|y_{\phi}\right\|_{H_{0}^{1}(\Omega)}+\left\|y_{\phi}\right\|_{C(\bar{\Omega})} \leq C\|\phi\|_{L^{p}(\Omega)} .
$$

In the paper, we also need the following assumption.

$(A 5)$ For a.e. $x \in \Omega$,

$$
\delta(x) \leq \delta_{0}:=\frac{\epsilon_{0}}{4 C \max \left\{1 ;|\Omega|^{1 / p}\right\}},
$$

where $|\Omega|$ is the volume of $\Omega$ and $C$ is positive constant which is given in (8).

We now state our main result

Theorem 1.1. Suppose that assumptions $(A 1)-(A 5)$ are satisfied. Then there exist a neighborhood $M_{1} \times \Lambda_{1}$ of $(\bar{\mu}, \bar{\lambda})$ and a neighborhood $Z_{1}=Y_{1} \times U_{1}$ of $(\bar{y}, \bar{u})$ such that for each $(\mu, \lambda) \in M_{1} \times \Lambda_{1}, P(\mu, \lambda)$ has a unique solution $z(\mu, \lambda)=$ $(y(\mu, \lambda), u(\mu, \lambda)) \in Z_{1}$ and the map $z(\cdot, \cdot)$ is Hölder continuous, that is, there exist positive constants $l_{1}$ and $l_{2}$ such that

$$
\begin{aligned}
& \left\|y\left(\mu^{1}, \lambda^{1}\right)-y\left(\mu^{2}, \lambda^{2}\right)\right\|_{Y}+\left\|u\left(\mu^{1}, \lambda^{1}\right)-u\left(\mu^{2}, \lambda^{2}\right)\right\|_{L^{p}(\Omega)} \\
\leq & l_{1}\left\|\mu^{1}-\mu^{2}\right\|_{M}^{\alpha / p}+l_{2}\left\|\lambda^{1}-\lambda^{2}\right\|_{\Lambda}^{1 / p}
\end{aligned}
$$

for all $\left(\mu^{i}, \lambda^{i}\right) \in M_{1} \times \Lambda_{1}$ with $i=1,2$. Here $\alpha=\min \left\{\alpha_{1}, \alpha_{2}\right\}$.

In order to prove Theorem 1.1 we will establish some auxiliary results which are provided in section 2. Section 3 contains the proof of Theorem 1.1. Section 4 is destined for some examples illustrating Theorem 1.1.

\section{Auxiliary Results}

In this section we will give some properties of the set-valued map $K: \Lambda \rightrightarrows Z$, where $K(\lambda)$ is defined by (4). We begin with the following important result on the continuity of solutions of PDEs, which is due to E. Casas who did the associated pionieering work (see [5, Theorem 2.1] and [6, Theorem 2.1]) . For complement, we provide here a brief proof.

Lemma 2.1. [5, Theorem 2.1] Assume that conditions (A1) and (A2) are satisfied. Then for each $\phi \in L^{p}(\Omega)$ equation (7) has a unique weak solution $y_{\phi} \in H_{0}^{1}(\Omega) \cap C(\bar{\Omega})$ which has the a priori estimate

$$
\left\|y_{\phi}\right\|_{H_{0}^{1}(\Omega)}+\left\|y_{\phi}\right\|_{C(\bar{\Omega})} \leq C\|\phi\|_{L^{p}(\Omega)},
$$

where $C$ is a constant independent of $\phi$, and if $\phi_{n} \rightarrow \phi$ weakly in $L^{p}(\Omega)$ then $y_{\phi_{n}} \rightarrow y_{\phi}$ strongly in $H_{0}^{1}(\Omega) \cap C(\bar{\Omega})$. Moreover, the maximum principle holds, that is,

$$
\phi \geq 0 \text { implies } y_{\phi} \geq 0 \text {. }
$$


Proof. Since $\phi \in L^{p}(\Omega) \hookrightarrow L^{2}(\Omega)$, the Lax-Milgram theorem and G. Stampacchia (see [7, Theorem 12.4]) imply that (7) has a unique solution $y_{\phi} \in H_{0}^{1}(\Omega) \cap L^{\infty}(\Omega)$, and there exists a constant $C_{1}>0$ independent of $\phi$ such that

$$
\left\|y_{\phi}\right\|_{H_{0}^{1}(\Omega)}+\left\|y_{\phi}\right\|_{L^{\infty}(\Omega)} \leq C_{1}\|\phi\|_{W^{-1, r}(\Omega)},
$$

where $2 \leq r<\frac{2 N}{N-2}$. The continuity of $y_{\phi}(\cdot)$ is followed from [9, Theorem 8.30]. Since the imbedding $L^{2}(\Omega) \hookrightarrow W^{-1, r}(\Omega)$ is compact, there exists a constant $C$ independent of $\phi$ such that (10) is satisfied. If $\phi_{n} \rightarrow \phi$ weakly in $L^{p}(\Omega)$ then $\phi_{n} \rightarrow \phi$ strongly in $W^{-1, r}(\Omega)$. Combining this with (11), we see that $y_{\phi_{n}} \rightarrow y_{\phi}$ strongly in $H_{0}^{1}(\Omega) \cap C(\bar{\Omega})$. Finally, by [2, Lemma 2.2], the maximum principle is proved.

From Lemma 2.1, we can define a linear continuous solution mapping

$$
\begin{aligned}
S: L^{p}(\Omega) & \rightarrow Y \\
\phi & \mapsto y,
\end{aligned}
$$

where $y$ is a unique solution of (7) corresponding to $\phi$.

Lemma 2.2. Under assumptions (A1), (A2) and (A5), for each $\lambda \in \Lambda, K(\lambda)$ is a nonempty and closed convex set in $Z$.

Proof. For each $\lambda=\left(\lambda_{1}, \lambda_{2}, \lambda_{3}\right) \in \Lambda$. Obviously, $K(\lambda)$ is convex. Now we show that $K(\lambda)$ is nonempty subset. In fact, we choose $u(x)=\max \left\{u_{0} ;-\lambda_{1}(x)\right\}$, where $u_{0}$ is given by

$$
u_{0}:=\max \left\{\frac{\epsilon_{0}|\Omega|^{-1 / p}\left\|\lambda_{1}\right\|_{L^{p}(\Omega)}+4\left\|\lambda_{3}\right\|_{L^{\infty}(\Omega)}}{3 \epsilon_{0}} ;\left\|\lambda_{2}\right\|_{L^{\infty}(\Omega)}\right\} .
$$

This implies $u+\lambda_{1}=\frac{1}{2}\left(u_{0}+\lambda_{1}+\left|u_{0}+\lambda_{1}\right|\right) \geq 0$ and $u \geq \lambda_{2}$ in $\Omega$.

Moreover, we set $y=S\left(u+\lambda_{1}\right)$ then $(y, u)$ satisfies (2) and $y \geq 0$.

From Lemma 2.1, we get

$$
\begin{aligned}
\|y\|_{C(\bar{\Omega})} & \leq C\left\|u+\lambda_{1}\right\|_{L^{p}(\Omega)} \\
& \leq C\left(\left\|u_{0}\right\|_{L^{p}(\Omega)}+\left\|\lambda_{1}\right\|_{L^{p}(\Omega)}\right) \\
& \leq C\left(u_{0}|\Omega|^{1 / p}+\left\|\lambda_{1}\right\|_{L^{p}(\Omega)}\right) .
\end{aligned}
$$

Combining this with $(A 5)$ yields

$$
\begin{aligned}
\delta y+\lambda_{3} & \leq \delta_{0}\|y\|_{C(\bar{\Omega})}+\left\|\lambda_{3}\right\|_{L^{\infty}(\Omega)} \\
& \leq \frac{\epsilon_{0}}{4 C|\Omega|^{1 / p}} C\left(u_{0}|\Omega|^{1 / p}+\left\|\lambda_{1}\right\|_{L^{p}(\Omega)}\right)+\left\|\lambda_{3}\right\|_{L^{\infty}(\Omega)} \\
& \leq \frac{\epsilon_{0} u_{0}}{4}+\frac{\epsilon_{0}\left\|\lambda_{1}\right\|_{L^{p}(\Omega)}}{4|\Omega|^{1 / p}}+\left\|\lambda_{3}\right\|_{L^{\infty}(\Omega)} .
\end{aligned}
$$


On the other hand, since $u \geq u_{0} \geq 0$ and $\epsilon \geq \epsilon_{0}>0$,

$$
\begin{aligned}
\epsilon u & \geq \epsilon_{0} u_{0} \\
& \geq \frac{\epsilon_{0} u_{0}}{4}+\frac{1}{4}\left(\epsilon_{0}|\Omega|^{-1 / p}\left\|\lambda_{1}\right\|_{L^{p}(\Omega)}+4\left\|\lambda_{3}\right\|_{L^{\infty}(\Omega)}\right) .
\end{aligned}
$$

Hence $\epsilon u \geq \delta y+\lambda_{3}$, and so $(y, u)$ satisfies (3). Consequently, $K(\lambda) \neq \emptyset$.

Finally we show that $K(\lambda)$ is closed.

Indeed. Assume that $z_{n}=\left(y_{n}, u_{n}\right) \in K(\lambda)$ and $z_{n} \rightarrow z=(y, u)$ in $Z$. Then $z_{n} \rightarrow$ $z$ in $L^{2}(\Omega) \times L^{p}(\Omega)$. By passing a subsequence where $z_{n} \rightarrow z$ as $n \rightarrow \infty$ a. e. in $\Omega$ (see, [4, Theorem 4.9, pp. 94]). In other words, there exists a subset $B$ which has measure zero such that

$$
z_{n}(x) \rightarrow z(x)=(y(x), u(x)) \text { for all } x \in \Omega \backslash B \quad \text { as } n \rightarrow \infty .
$$

Since

$$
\begin{cases}u_{n} \geq \lambda_{2} & \text { in } \Omega, \\ \epsilon u_{n} \geq \delta y_{n}+\lambda_{3} & \text { in } \Omega,\end{cases}
$$

there exists subset $P_{n}$ which has measure zero such that

$$
\begin{cases}u_{n}(x) \geq \lambda_{2}(x) & \text { for all } x \in \Omega \backslash P_{n}, \\ \epsilon(x) u_{n}(x) \geq \delta(x) y_{n}(x)+\lambda_{3}(x) & \text { for all } x \in \Omega \backslash P_{n} .\end{cases}
$$

Setting $T=\bigcup_{n \geq 1} P_{n} \cup B$, we see that $T$ has measure zero. Letting $n \rightarrow \infty$, we obtain from the above that

$$
\begin{cases}u(x) \geq \lambda_{2}(x) & \text { for all } x \in \Omega \backslash T, \\ \epsilon(x) u(x) \geq \delta(x) y(x)+\lambda_{3}(x) & \text { for all } x \in \Omega \backslash T .\end{cases}
$$

Hence $z=(y, u)$ satisfies (3). It remains to prove that $(y, u)$ satisfies (2). In fact, we set $\bar{y}=S\left(u+\lambda_{1}\right)$. We then have

$$
\begin{aligned}
\|y-\bar{y}\|_{Y} & \leq\left\|y-y_{n}\right\|_{Y}+\left\|y_{n}-\bar{y}\right\|_{Y} \\
& \leq\left\|y-y_{n}\right\|_{Y}+\left\|S\left(u_{n}+\lambda_{1}\right)-S\left(u+\lambda_{1}\right) \mid\right\|_{Y} \\
& \leq\left\|y-y_{n}\right\|_{Y}+C\left\|u_{n}-u\right\|_{L^{p}(\Omega)} .
\end{aligned}
$$

Letting $n \rightarrow \infty$, we obtain $y=\bar{y}$. Consequently, $(y, u)$ satisfies (2) and $(y, u) \in K(\lambda)$. Hence $K(\lambda)$ is closed. The proof of the lemma is complete.

Lemma 2.3. Under assumptions of Lemma 2.2, the set-valued map $K: \Lambda \rightrightarrows Z$ is Lipschitz continuous, that is, there exists a positive constant $k$ such that

$$
K(\lambda) \subset K(\beta)+k\|\lambda-\beta\|_{\Lambda} \bar{B}_{Z}, \quad \forall \lambda, \beta \in \Lambda .
$$


Proof. Take any $\lambda=\left(\lambda_{1}, \lambda_{2}, \lambda_{3}\right), \beta=\left(\beta_{1}, \beta_{2}, \beta_{3}\right) \in \Lambda$. For convenience we put

$$
\begin{aligned}
& \gamma=\|\lambda-\beta\|_{\Lambda}=\left\|\lambda_{1}-\beta_{1}\right\|_{L^{p}(\Omega)}+\left\|\lambda_{2}-\beta_{2}\right\|_{L^{\infty}(\Omega)}+\left\|\lambda_{3}-\beta_{3}\right\|_{L^{\infty}(\Omega)}, \\
& \tau(x)=\gamma+\left|\lambda_{1}(x)-\beta_{1}(x)\right|, \quad \theta=\max \left\{1 ; \frac{4+\epsilon_{0}}{2 \epsilon_{0}}\right\} .
\end{aligned}
$$

Taking any $z_{\lambda}=\left(y_{\lambda}, u_{\lambda}\right) \in K(\lambda)$, we choose $u_{\beta}=u_{\lambda}+\theta \tau$ and set $y_{\beta}=S\left(u_{\beta}+\beta_{1}\right)$ is a unique solution to the following elliptic equation

$$
\begin{cases}A y=u_{\beta}+\beta_{1} & \text { in } \Omega, \\ y=0 & \text { on } \partial \Omega .\end{cases}
$$

Since $u_{\lambda} \geq \lambda_{2}$, we have

$$
u_{\beta} \geq \beta_{2} \text {. }
$$

\section{Moreover}

$$
\begin{aligned}
y_{\beta}=S\left(u_{\beta}+\beta_{1}\right) & =S\left(u_{\lambda}+\lambda_{1}\right)+S\left(\theta \tau+\beta_{1}-\lambda_{1}\right) \\
& =y_{\lambda}+\sigma,
\end{aligned}
$$

where $\sigma=S\left(\theta \tau+\beta_{1}-\lambda_{1}\right)$. Since $\theta \tau+\beta_{1}-\lambda_{1} \geq 0$, Lemma 2.1 implies that $\sigma \geq 0$. Hence

$$
\begin{aligned}
& \epsilon u_{\beta}-\delta y_{\beta}-\beta_{3} \\
= & \epsilon u_{\lambda}-\delta y_{\lambda}-\lambda_{3}+\theta \epsilon \tau-\delta \sigma+\lambda_{3}-\beta_{3} \\
\geq & \theta \epsilon_{0} \tau-\delta_{0}\|\sigma\|_{C(\bar{\Omega})}-\left\|\lambda_{3}-\beta\right\|_{L^{\infty}(\Omega)} \quad\left(\text { because of } \epsilon u_{\lambda}-\delta y_{\lambda}-\lambda_{3} \geq 0\right) \\
\geq & \theta \epsilon_{0} \tau-\delta_{0} C\left\|\theta \tau+\beta_{1}-\lambda_{1}\right\|_{L^{p}(\Omega)}-\left\|\lambda_{3}-\beta_{3}\right\|_{L^{\infty}(\Omega)} \\
\geq & \theta \epsilon_{0} \tau-\delta_{0} C\left[\theta\|\tau\|_{L^{p}(\Omega)}+\left\|\lambda_{1}-\beta_{1}\right\|_{L^{p}(\Omega)}\right]-\left\|\lambda_{3}-\beta_{3}\right\|_{L^{\infty}(\Omega)} .
\end{aligned}
$$

This implies

$$
\begin{aligned}
& \epsilon u_{\beta}-\delta y_{\beta}-\beta_{3} \\
\geq & \theta \epsilon_{0} \tau-\delta_{0} C\left[\theta|\Omega|^{1 / p} \gamma+(\theta+1)\left\|\lambda_{1}-\beta_{1}\right\|_{L^{p}(\Omega)}\right]-\left\|\lambda_{3}-\beta_{3}\right\|_{L^{\infty}(\Omega)} \\
\geq & \theta \epsilon_{0} \tau-\frac{1}{4} \theta \epsilon_{0} \gamma-\frac{1}{4}(\theta+1) \epsilon_{0}\left\|\lambda_{1}-\beta_{1}\right\|_{L^{p}(\Omega)}-\left\|\lambda_{3}-\beta_{3}\right\|_{L^{\infty}(\Omega)} \\
\geq & \theta \epsilon_{0} \tau-\frac{1}{4} \epsilon_{0}(2 \theta+1) \gamma-\left\|\lambda_{3}-\beta_{3}\right\|_{L^{\infty}(\Omega)} \\
\geq & \left.\left(\theta \epsilon_{0}-1\right) \gamma-\frac{1}{4} \epsilon_{0}(2 \theta+1) \gamma \quad \text { (because of } \tau \geq \gamma \text { and }\left\|\lambda_{3}-\beta_{3}\right\|_{L^{\infty}(\Omega)} \leq \gamma\right) \\
\geq & \frac{1}{4}\left(2 \theta \epsilon_{0}-4-\epsilon_{0}\right) \gamma \geq 0 .
\end{aligned}
$$


Combining this with (13) yields $z_{\beta}=\left(y_{\beta}, u_{\beta}\right)$ satisfying (3). This implies $z_{\beta} \in K(\beta)$. On the other hand, we have

$$
\begin{aligned}
\left\|u_{\beta}-u_{\lambda}\right\|_{L^{p}(\Omega)}=\|\theta \tau\|_{L^{p}(\Omega)} & \leq \theta|\Omega|^{1 / p} \gamma+\theta\left\|\beta_{1}-\lambda_{1}\right\|_{L^{p}(\Omega)} \\
& \leq \theta\left(|\Omega|^{1 / p}+1\right) \gamma .
\end{aligned}
$$

By Lemma 2.1,

$$
\begin{aligned}
\left\|y_{\beta}-y_{\lambda}\right\|_{Y}=\|\sigma\|_{Y} & \leq C\left\|\theta \tau+\left(\beta_{1}-\lambda_{1}\right)\right\|_{L^{p}(\Omega)} \\
& \leq C\left(\theta\|\tau\|_{L^{p}(\Omega)}+\left\|\beta_{1}-\lambda_{1}\right\|_{L^{p}(\Omega)}\right) \\
& \leq C\left(\theta|\Omega|^{1 / p} \gamma+(\theta+1)\left\|\beta_{1}-\lambda_{1}\right\|_{L^{p}(\Omega)}\right) \\
& \leq C\left(\theta|\Omega|^{1 / p}+(\theta+1)\right) \gamma .
\end{aligned}
$$

Combining (14) with (15) we have the following inequality

$$
\left\|y_{\beta}-y_{\lambda}\right\|_{Y}+\left\|u_{\beta}-u_{\lambda}\right\|_{L^{p}(\Omega)} \leq k \gamma,
$$

where $k=\theta\left(|\Omega|^{1 / p}+1\right)+C\left(\theta|\Omega|^{1 / p}+(\theta+1)\right)$. The proof is complete.

\section{Proof of the Main Result}

From Lemma 2.3 , we get

$$
K(\bar{\lambda}) \subset K(\lambda)+k\|\bar{\lambda}-\lambda\|_{\Lambda} \bar{B}_{Z}, \quad \forall \lambda \in \Lambda .
$$

Fix $r_{0}>0$ such that $k r_{0} \leq \epsilon_{2}$, where $\epsilon_{2}$ is given in the assumption $(A 3)$. Then we have

$$
K(\lambda) \cap\left(\bar{z}+\epsilon_{2} \bar{B}_{Z}\right) \neq \emptyset, \quad \forall \lambda \in \bar{B}_{\Lambda}\left(\bar{\lambda}, r_{0}\right)
$$

Let us put

$$
\begin{gathered}
Y_{0}=\bar{B}_{Y}\left(\bar{y}, \epsilon_{2}\right), U_{0}=\bar{B}_{U}\left(\bar{u}, \epsilon_{2}\right), Z_{0}=Y_{0} \times U_{0}, \\
M_{0}=\bar{B}_{M}\left(\bar{\mu}, \epsilon_{1}\right) \text { and } \Lambda_{0}=\bar{B}_{\Lambda}\left(\bar{\lambda}, r_{0}\right) .
\end{gathered}
$$

Easily, we see that

$$
\bar{B}_{Z}\left(\bar{z}, \epsilon_{2}\right) \subset Z_{0} .
$$

Combining this with (16) yields

$$
K(\lambda) \cap Z_{0} \neq \emptyset \quad \forall \lambda \in \Lambda_{0} .
$$


Lemma 3.1. Suppose that assumptions $(A 1)-(A 5)$ are fulfilled. Then the following assertions hold:

(i) For each $\mu \in M_{0}$, the function $F(\cdot, \mu)$ is Gâteaux differentiable and its derivative is given by

$$
\begin{aligned}
\left\langle F_{z}(z, \mu), h\right\rangle & =\left\langle F_{y}(y, u, \mu), h_{1}\right\rangle+\left\langle F_{u}(y, u, \mu), h_{2}\right\rangle \\
& =\int_{\Omega} f_{y}(x, y(x), u(x), \mu(x)) h_{1}(x) d x+\int_{\Omega} f_{u}(x, y(x), u(x), \mu(x)) h_{2}(x) d x,
\end{aligned}
$$

for all $h=\left(h_{1}, h_{2}\right) \in Z$. Moreover, $F_{z}(\cdot, \cdot)$ is uniformly bounded on $Z_{0} \times M_{0}$.

(ii) There exists a positive constant $l_{0}$ such that

$$
\left\|F_{z}\left(z, \mu^{1}\right)-F_{z}\left(z, \mu^{2}\right)\right\|_{Z^{*}} \leq l_{0}\left\|\mu^{1}-\mu^{2}\right\|_{M}^{\alpha}, \quad \forall z \in Z_{0}, \mu^{1}, \mu^{2} \in M_{0} .
$$

(iii) $F_{z}(\cdot, \mu)$ is strongly monotone, that is

$$
\left\langle F_{z}\left(z_{1}, \mu\right)-F_{z}\left(z_{2}, \mu\right), z_{1}-z_{2}\right\rangle \geq \rho\left\|u_{1}-u_{2}\right\|_{L^{p}(\Omega)}^{p} \quad \forall z_{1}, z_{2} \in Z_{0},
$$

where $z_{1}=\left(y_{1}, u_{1}\right)$ and $z_{2}=\left(y_{2}, z_{2}\right)$.

Proof. By $(A 4)$, for each $\mu \in M_{0}$, the first variation $F_{z}(z, \mu)(h)$ of $F(\cdot, \mu)$ at a point $z=(y, u) \in Z$ does exist and defined by

$$
\begin{aligned}
F_{z}(z, \mu)(h) & =\left\langle F_{z}(z, \mu), h\right\rangle \\
& =\left\langle F_{y}(y, u, \mu), h_{1}\right\rangle+\left\langle F_{u}(y, u, \mu), h_{2}\right\rangle \\
& =\int_{\Omega} f_{y}(x, y(x), u(x), \mu(x)) h_{1}(x) d x+\int_{\Omega} f_{u}(x, y(x), u(x), \mu(x)) h_{2}(x) d x,
\end{aligned}
$$

for all $h=\left(h_{1}, h_{2}\right) \in Z$. Obviously, $F_{z}(z, \mu)(\cdot)$ is a linear mapping. We now show that $F_{z}(\cdot, \cdot)$ is uniformly bounded on $Z_{0} \times M_{0}$.

Indeed. For any $z=(y, u) \in Z_{0}$, we have

$$
\left\|F_{z}(z, \bar{\mu})\right\|_{Z^{*}} \leq\left\|F_{y}(y, u, \bar{\mu})\right\|_{Y^{*}}+\left\|F_{u}(y, u, \bar{\mu})\right\|_{L^{q}(\Omega)},
$$

where $q$ is the conjugate number of $p$.

By the Hölder inequality, there exist constants $c_{j}>0, j=1,2$ such that

$$
\begin{gathered}
\int_{\Omega}|u|^{s}\left|h_{1}\right| d x \leq c_{1}\|u\|_{L^{p}(\Omega)}^{s}\left\|h_{1}\right\|_{C(\bar{\Omega})} \leq c_{1}\|u\|_{L^{p}(\Omega)}^{s}\left\|h_{1}\right\|_{Y} \quad \forall h_{1} \in Y, \\
\text { (22) and } \quad \int_{\Omega}|u|^{d}\left|h_{2}\right| d x \leq c_{2}\|u\|_{L^{p}(\Omega)}^{d}\left\|h_{2}\right\|_{L^{p}(\Omega)} \quad \forall h_{2} \in L^{p}(\Omega), \\
\text { where } 0 \leq s \leq p \text { and } 0 \leq d \leq p-1 .
\end{gathered}
$$


By definitions of $H_{i}(i=1,2)$ and (22), there exist positive constants $C_{H_{i}}$ such that

$$
\int_{\Omega} H_{1}(|u|)\left|h_{1}\right| d x \leq C_{H_{1}} H_{1}\left(\|u\|_{L^{p}(\Omega)}\right)\left\|h_{1}\right\|_{Y} \quad \forall h_{1} \in Y
$$

and

$$
\int_{\Omega} H_{2}(|u|)\left|h_{2}\right| d x \leq C_{H_{2}} H_{2}\left(\|u\|_{L^{p}(\Omega)}\right)\left\|h_{2}\right\|_{L^{p}(\Omega)} \quad \forall h_{2} \in L^{p}(\Omega) .
$$

We put

$$
A_{i}=\max \left\{a_{i}\left(x,\left|t_{1}\right|,\left|t_{2}\right|\right):\left(x, t_{1}, t_{2}\right) \in \bar{\Omega} \times\left[0, \delta_{1}\right] \times\left[0, \delta_{2}\right]\right\}, i=1,2,
$$

where $\delta_{1}:=\|\bar{y}\|_{C(\bar{\Omega})}+\epsilon_{2}, \delta_{2}:=\|\bar{\mu}\|_{M}+\epsilon_{1}$.

By $(A 4)$,

$$
\begin{aligned}
\left\|F_{y}(y, u, \bar{\mu})\right\|_{Y^{*}} & =\sup \left\{\left\langle F_{y}(y, u, \bar{\mu}), h_{1}\right\rangle: h_{1} \in Y,\left\|h_{1}\right\|_{Y} \leq 1\right\} \\
& =\sup \left\{\int_{\Omega} f_{y}(x, y(x), u(x), \bar{\mu}(x)) h_{1}(x) d x:\left\|h_{1}\right\|_{Y} \leq 1\right\} \\
& \leq \sup \left\{\int_{\Omega} a_{1}(\cdot,|y|,|\bar{\mu}|) H_{1}(|u|)\left|h_{1}\right| d x:\left\|h_{1}\right\|_{Y} \leq 1\right\} \\
& \leq A_{1} \sup \left\{\int_{\Omega} H_{1}(|u|)\left|h_{1}\right| d x:\left\|h_{1}\right\|_{Y} \leq 1\right\} .
\end{aligned}
$$

Combining (23) with (25) yields

$$
\begin{aligned}
\left\|F_{y}(y, u, \bar{\mu})\right\|_{Y^{*}} & \leq A_{1} C_{H_{1}} \sup \left\{H_{1}\left(\|u\|_{L^{p}(\Omega)}\right)\left\|h_{1}\right\|_{Y}:\left\|h_{1}\right\|_{Y} \leq 1\right\} \\
& \leq A_{1} C_{H_{1}} H_{1}\left(\|u\|_{L^{p}(\Omega)}\right) \\
& \leq A_{1} C_{H_{1}} H_{1}\left(\|\bar{u}\|_{L^{p}(\Omega)}+\epsilon_{2}\right) .
\end{aligned}
$$

Using similar arguments, we obtain

$$
\left\|F_{u}(y, u, \bar{\mu})\right\|_{L^{q}(\Omega)} \leq A_{2} C_{H_{2}} H_{2}\left(\|\bar{u}\|_{L^{p}(\Omega)}+\epsilon_{2}\right) .
$$

Combining (21) with (26) and (27) we conclude that

$$
\left\|F_{z}(z, \bar{\mu})\right\|_{Z^{*}} \leq A_{1} C_{H_{1}} H_{1}\left(\|\bar{u}\|_{L^{p}(\Omega)}+\epsilon_{2}\right)+A_{2} C_{H_{2}} H_{2}\left(\|\bar{u}\|_{L^{p}(\Omega)}+\epsilon_{2}\right) .
$$

On the other hand, for any $z=(y, u) \in Z_{0}$ and $\mu^{1}, \mu^{2} \in M_{0}$, we have

$$
\begin{aligned}
& \left\|F_{z}\left(z, \mu^{1}\right)-F_{z}\left(z, \mu^{2}\right)\right\|_{Z^{*}} \\
& \quad \leq\left\|F_{y}\left(y, u, \mu^{1}\right)-F_{y}\left(y, u, \mu^{2}\right)\right\|_{Y^{*}}+\left\|F_{u}\left(y, u, \mu^{1}\right)-F_{u}\left(y, u, \mu^{2}\right)\right\|_{L^{q}(\Omega)} .
\end{aligned}
$$


In the same manner, using $(A 4)$, we get

$$
\begin{aligned}
\left\|F_{y}\left(y, u, \mu^{1}\right)-F_{y}\left(y, u, \mu^{2}\right)\right\|_{Y^{*}} & \leq B_{1} C_{H_{1}} H_{1}\left(\|u\|_{L^{p}(\Omega)}\right)\left\|\mu^{1}-\mu^{2}\right\|_{M}^{\alpha_{1}} \\
& \leq B_{1} C_{H_{1}} H_{1}\left(\|\bar{u}\|_{L^{p}(\Omega)}+\epsilon_{2}\right)\left\|\mu^{1}-\mu^{2}\right\|_{M}^{\alpha_{1}}
\end{aligned}
$$

and

$$
\begin{aligned}
\left\|F_{u}\left(y, u, \mu^{1}\right)-F_{u}\left(y, u, \mu^{2}\right)\right\|_{L^{q}} & \leq B_{2} C_{H_{2}} H_{2}\left(\|u\|_{L^{p}(\Omega)}\right)\left\|\mu^{1}-\mu^{2}\right\|_{M}^{\alpha_{2}} \\
& \leq B_{2} C_{H_{2}} H_{2}\left(\|\bar{u}\|_{L^{p}(\Omega)}+\epsilon_{2}\right)\left\|\mu^{1}-\mu^{2}\right\|_{M}^{\alpha_{2}},
\end{aligned}
$$

where $B_{i}:=\max \left\{b_{i}\left(x, t_{1}, t_{2}, t_{3}\right):\left(x, t_{1}, t_{2}, t_{3}\right) \in \bar{\Omega} \times\left[0, \delta_{1}\right] \times\left[0, \delta_{2}\right]^{2}\right\}, i=1,2$. From (29)-(31) we deduce that

$$
\left\|F_{z}\left(z, \mu^{1}\right)-F_{z}\left(z, \mu^{2}\right)\right\|_{Z^{*}} \leq l_{0}\left\|\mu^{1}-\mu^{2}\right\|_{M}^{\alpha},
$$

where $l_{0}:=B_{1} C_{H_{1}} H_{1}\left(\|\bar{u}\|_{L^{p}(\Omega)}+\epsilon_{2}\right)\left(2 \epsilon_{1}\right)^{\alpha_{1}-\alpha}+B_{2} C_{H_{2}} H_{2}\left(\|\bar{u}\|_{L^{p}(\Omega)}+\epsilon_{2}\right)\left(2 \epsilon_{1}\right)^{\alpha_{2}-\alpha}$. We obtain assertion $(i i)$.

Since (28) and (32), we get

$$
\begin{aligned}
\left\|F_{z}(z, \mu)\right\|_{Z^{*}} & \leq\left\|F_{z}(z, \mu)-F_{z}(z, \bar{\mu})\right\|_{Z^{*}}+\left\|F_{z}(z, \bar{\mu})\right\|_{Z^{*}} \\
& \leq l_{0}\|\mu-\bar{\mu}\|_{M}^{\alpha}+A_{1} C_{H_{1}} H_{1}\left(\|\bar{u}\|_{L^{p}(\Omega)}+\epsilon_{2}\right)+A_{2} C_{H_{2}} H_{2}\left(\|\bar{u}\|_{L^{p}(\Omega)}+\epsilon_{2}\right) .
\end{aligned}
$$

Hence

$$
\left\|F_{z}(z, \mu)\right\|_{Z^{*}} \leq l
$$

where $l=l_{0} \epsilon_{1}^{\alpha}+A_{1} C_{H_{1}} H_{1}\left(\|\bar{u}\|_{L^{p}(\Omega)}+\epsilon_{2}\right)+A_{2} C_{H_{2}} H_{2}\left(\|\bar{u}\|_{L^{p}(\Omega)}+\epsilon_{2}\right)$. This implies that $F_{z}(\cdot, \cdot)$ is uniformly bounded on $Z_{0} \times M_{0}$. Consequently, the function $F(\cdot, \mu)$ is Gâteaux differentiable for all $\mu \in M_{0}$. Hence, assertion $(i)$ is obtained.

Fix any $\mu \in M_{0}$. Taking any $z_{i}=\left(y_{i}, u_{i}\right) \in Z_{0}, i=1,2$, we have

$$
\begin{aligned}
& \left\langle F_{z}\left(z_{1}, \mu\right)-F_{z}\left(z_{2}, \mu\right), z_{1}-z_{2}\right\rangle \\
= & \int_{\Omega}\left(f_{z}\left(x, z_{1}(x), \mu(x)\right)-f_{z}\left(x, z_{2}(x), \mu(x)\right)\left(z_{1}(x)-z_{2}(x)\right) d x .\right.
\end{aligned}
$$

From this and $(A 3)$ we obtain

$$
\left\langle F_{z}\left(z_{1}, \mu\right)-F_{z}\left(z_{2}, \mu\right), z_{1}-z_{2}\right\rangle \geq \rho\left\|u_{1}-u_{2}\right\|_{L^{p}(\Omega)}^{p} .
$$

The proof of the lemma is complete. 
Lemma 3.2. Under assumptions of Lemma 3.1, for each $(\mu, \lambda) \in M_{0} \times \Lambda_{0}$, the problem

$$
P_{0}(\mu, \lambda) \quad\left\{\begin{array}{l}
F(z, \mu) \rightarrow \inf \\
z \in K(\lambda) \cap Z_{0}
\end{array}\right.
$$

has a unique solution.

Proof. Put

$$
\xi=\inf \left\{F(z, \mu): z \in K(\lambda) \cap Z_{0}\right\} .
$$

Then there exists a sequence $z_{n}=\left(y_{n}, u_{n}\right) \in K(\lambda) \cap Z_{0}$ such that

$$
\xi=\lim _{n \rightarrow \infty} F\left(z_{n}, \mu\right) .
$$

Since $\left\{u_{n}\right\}$ is bounded and $L^{p}(\Omega)$ is a reflexive Banach space, we can assume that

$$
u_{n} \rightarrow \hat{u} \quad \text { in } \quad L^{p}(\Omega) .
$$

By Lemma 2.1, we get

$$
y_{n} \rightarrow \hat{y} \quad \text { in } \quad Y
$$

for some $\hat{z}=(\hat{y}, \hat{u}) \in Y \times L^{p}(\Omega)$. By Lemma $2.2, K(\lambda)$ is a weakly closed set. Consequently, $\hat{z}=(\hat{y}, \hat{u}) \in K(\lambda)$. Since $Z_{0}$ is a weakly closed subset, $\hat{z} \in Z_{0}$. Thus we get $\hat{z} \in K(\lambda) \cap Z_{0}$ and

$$
F(\hat{z}, \mu) \geq \xi
$$

On the other hand, by a property of convex functions, we have

$$
\begin{aligned}
& f\left(x, y_{n}(x), u_{n}(x), \mu(x)\right) \geq f(x, \hat{y}(x), \hat{u}(x), \mu(x)) \\
& +\left\langle f_{y}(x, \hat{y}(x), \hat{u}(x), \mu(x)), y_{n}(x)-\hat{y}(x)\right\rangle+\left\langle f_{u}(x, \hat{y}(x), \hat{u}(x), \mu(x)), u_{n}(x)-\hat{u}(x)\right\rangle .
\end{aligned}
$$

It follows that

$$
\begin{aligned}
F\left(y_{n}, u_{n}, \mu\right) \geq F(\hat{y}, \hat{u}, \mu) & +\int_{\Omega} f_{y}(x, \hat{y}(x), \hat{u}(x), \mu(x))\left(y_{n}(x)-\hat{y}(x)\right) d x \\
& +\int_{\Omega} f_{u}(x, \hat{y}(x), \hat{u}(x), \mu(x))\left(u_{n}(x)-\hat{u}(x)\right) d x .
\end{aligned}
$$

By $(A 2)$ and $(A 4)$ we can show that $f_{y}(\cdot, \hat{y}, \hat{u}, \mu) \in L^{2}(\Omega)$ and $f_{u}(\cdot, \hat{y}, \hat{u}, \mu) \in L^{q}(\Omega)$. Letting $n \rightarrow \infty$, we obtain from the above that $\xi \geq F(\hat{y}, \hat{u}, \mu)$. Combining this with (34) we have $\xi=F(\hat{y}, \hat{u}, \mu)$.

We now prove that $\xi$ is finite. To do this we first show that $F(\bar{z}, \cdot)$ is bounded on $M_{0}$. In fact, for any $\mu \in M_{0}$ from $(A 2)$, we get 


$$
\begin{aligned}
|F(\bar{z}, \mu)-F(\bar{z}, \bar{\mu})| & \leq \int_{\Omega}|f(x, \bar{y}(x), \bar{u}(x), \mu(x))-f(x, \bar{y}(x), \bar{u}(x), \bar{\mu}(x))| d x \\
& \leq \int_{\Omega} g(x,|\bar{y}(x)|,|\mu(x)|,|\bar{\mu}(x)|) H_{1}(|\bar{u}(x)|) d x \\
& \leq \eta \int_{\Omega} H_{1}(|\bar{u}(x)|) d x \\
& \leq \eta C_{H_{1}} H_{1}\left(\|\bar{u}\|_{L^{p}(\Omega)}\right)
\end{aligned}
$$

where

$\eta=\max \left\{g\left(x, t_{1}, t_{2}, t_{3}\right):\left(x, t_{1}, t_{2}, t_{3}\right) \in \bar{\Omega} \times\left[0,\|\bar{y}\|_{C(\bar{\Omega})}\right] \times\left[0,\|\bar{\mu}\|_{M}+\epsilon_{1}\right] \times\left[0,\|\bar{\mu}\|_{M}\right]\right\}$.

Consequently,

$$
|F(\bar{z}, \mu)-F(\bar{z}, \bar{\mu})| \leq \eta C_{H_{1}} H_{1}\left(\|\bar{u}\|_{L^{p}(\Omega)}\right) .
$$

We obtain the desired conclusion.

From (35), the uniform boundedness of $F_{z}(\cdot, \cdot)$ on $Z_{0} \times M_{0}$ and the mean value theorem, for all $\mu \in M_{0}$, we get

$$
\begin{aligned}
|F(\hat{z}, \mu)-F(\bar{z}, \bar{\mu})| & \leq|F(\hat{z}, \mu)-F(\bar{z}, \mu)|+|F(\bar{z}, \mu)-F(\bar{z}, \bar{\mu})| \\
& \leq \sup _{0 \leq t \leq 1}\left\|F_{z}(\bar{z}+t(\hat{z}-\bar{z}), \mu)\right\|_{Z^{*}}\|\hat{z}-\bar{z}\|_{Z}+|F(\bar{z}, \mu)-F(\bar{z}, \bar{\mu})| \\
& \leq \sup _{z^{\prime} \in Z_{0}}\left\|F_{z}\left(z^{\prime}, \mu\right)\right\|_{Z^{*}}\|\hat{z}-\bar{z}\|_{Z}+\eta C_{H_{1}} H_{1}\left(\|\bar{u}\|_{L^{p}(\Omega)}\right) \\
& <+\infty .
\end{aligned}
$$

This implies that $|F(\hat{z}, \mu)|<+\infty$ and so $\xi$ is finite.

It remains to show that problem $P_{0}(\mu, \lambda)$ has a unique solution. Indeed, we assume that $z_{i}(\mu, \lambda)=\left(y_{i}(\mu, \lambda), u_{i}(\mu, \lambda)\right), i=1,2$ are solutions of $P_{0}(\mu, \lambda)$. It follows that

$$
\left\langle F_{z}\left(z_{i}(\mu, \lambda), \mu\right), z-z_{i}(\mu, \lambda)\right\rangle \geq 0 \quad \forall z \in K(\lambda) \cap Z_{0}, i=1,2 .
$$

Hence

$$
\left\langle F_{z}\left(z_{1}(\mu, \lambda), \mu\right)-F_{z}\left(z_{2}(\mu, \lambda), \mu\right), z_{1}(\mu, \lambda)-z_{2}(\mu, \lambda)\right\rangle \leq 0 .
$$

From this and (iii) of Lemma 3.1, we get

$$
\begin{aligned}
0 & \geq\left\langle F_{z}\left(z_{1}(\mu, \lambda), \mu\right)-F_{z}\left(z_{2}(\mu, \lambda), \mu\right), z_{1}(\mu, \lambda)-z_{2}(\mu, \lambda)\right\rangle \\
& \geq \rho\left\|u_{1}(\mu, \lambda)-u_{2}(\mu, \lambda)\right\|_{L^{p}(\Omega)}^{p} .
\end{aligned}
$$

It follows $u_{1}(\mu, \lambda)=u_{2}(\mu, \lambda)$. Since $y_{i}(\mu, \lambda)=S\left(u_{i}(\mu, \lambda)+\lambda_{1}\right)$ and Lemma 2.1, we obtain $y_{1}(\mu, \lambda)=y_{2}(\mu, \lambda)$. Hence $z_{1}(\mu, \lambda)=z_{2}(\mu, \lambda)$. This proves the lemma. 
Proof of Theorem 1.1. For each $(\mu, \lambda) \in M_{0} \times \Lambda_{0}$, due to Lemma 3.2, problem $P_{0}(\mu, \lambda)$ has a unique solution $z(\mu, \lambda)=(y(\mu, \lambda), u(\mu, \lambda)) \in K(\lambda) \cap Z_{0}$. Since $P_{0}(\mu, \lambda)$ is a convex problem, it must hold

$$
0 \in F_{z}(z(\mu, \lambda), \mu)+N\left(z(\mu, \lambda) ; K(\lambda) \cap Z_{0}\right) .
$$

It is equivalent to the variational inequality

$$
\left\langle F_{z}(z(\mu, \lambda), \mu), z-z(\mu, \lambda)\right\rangle \geq 0 \quad \forall z \in K(\lambda) \cap Z_{0}, \mu \in M_{0}, \lambda \in \Lambda_{0} .
$$

We first show that the solution mapping $z(\cdot, \cdot)$ is continuous at $(\bar{\mu}, \bar{\lambda})$.

In fact, fix any $(\mu, \lambda) \in M_{0} \times \Lambda_{0}$. By the Lipschitz continuous property of $K(\cdot)$, there exists an element $z_{1} \in K(\bar{\lambda})$ such that

$$
\left\|z(\mu, \lambda)-z_{1}\right\|_{Z} \leq k\|\lambda-\bar{\lambda}\|_{\Lambda} \leq \epsilon_{2} .
$$

Putting $\lambda=\bar{\lambda}$ and $\beta=\lambda$ in (12), we see that there exists $z_{2} \in K(\lambda)$ such that

$$
\left\|\bar{z}-z_{2}\right\|_{Z} \leq k\|\bar{\lambda}-\lambda\|_{\Lambda} \leq \epsilon_{2} .
$$

Since $\bar{z}$ and $z(\mu, \lambda)$ are solutions of $P(\bar{\mu}, \bar{\lambda})$ and $P(\mu, \lambda)$, respectively, it follows that

$$
\left\langle F_{z}(\bar{z}, \bar{\mu}), z_{1}-\bar{z}\right\rangle \geq 0 \quad \text { and } \quad\left\langle F_{z}(z(\mu, \lambda), \mu), z_{2}-z(\mu, \lambda)\right\rangle \geq 0 \text {. }
$$

By (ii) and (iii) of Lemma 3.1, and using (33), we have

$$
\begin{aligned}
& \rho\|u(\mu, \lambda)-\bar{u}\|_{L^{p}(\Omega)}^{p} \\
\leq & \left\langle F_{z}(z(\mu, \lambda), \mu)-F_{z}(\bar{z}, \mu), z(\mu, \lambda)-\bar{z}\right\rangle \\
\leq & \left\langle F_{z}(z(\mu, \lambda), \mu)-F_{z}(\bar{z}, \mu), z(\mu, \lambda)-\bar{z}\right\rangle+\left\langle F_{z}(\bar{z}, \bar{\mu}), z_{1}-\bar{z}\right\rangle \\
& +\left\langle F_{z}(z(\mu, \lambda), \mu), z_{2}-z(\mu, \lambda)\right\rangle \\
= & \left\langle F_{z}(z(\mu, \lambda), \mu), z_{2}-\bar{z}\right\rangle+\left\langle F_{z}(\bar{z}, \mu), z_{1}-z(\mu, \lambda)\right\rangle \\
& +\left\langle F_{z}(\bar{z}, \bar{\mu})-F_{z}(\bar{z}, \mu), z_{1}-\bar{z}\right\rangle \\
\leq & \left\|F_{z}(z(\mu, \lambda), \mu)\right\|_{Z^{*}}\left\|z_{2}-\bar{z}\right\|_{Z}+\left\|F_{z}(\bar{z}, \mu)\right\|_{Z^{*}}\left\|z_{1}-z(\mu, \lambda)\right\|_{Z} \\
& +\left\|F_{z}(\bar{z}, \bar{\mu})-F_{z}(\bar{z}, \mu)\right\|_{Z^{*}}\left\|z_{1}-\bar{z}\right\|_{Z} \\
\leq & 2 l k\|\lambda-\bar{\lambda}\|_{\Lambda}+l_{0}\left\|z_{1}-\bar{z}\right\|_{Z}\|\mu-\bar{\mu}\|_{M}^{\alpha} .
\end{aligned}
$$

On the other hand

$$
\left\|z_{1}-\bar{z}\right\|_{Z} \leq\left\|z_{1}-z(\mu, \lambda)\right\|_{Z}+\|z(\mu, \lambda)-\bar{z}\|_{Z} \leq \epsilon_{2}+2 \epsilon_{2}=3 \epsilon_{2} .
$$


Hence (38) implies that

$$
\|u(\mu, \lambda)-\bar{u}\|_{L^{p}(\Omega)}^{p} \leq \frac{2 l k}{\rho}\|\lambda-\bar{\lambda}\|_{\Lambda}+\frac{3 \epsilon_{2} l_{0}}{\rho}\|\mu-\bar{\mu}\|_{M}^{\alpha}
$$

From Lemma 2.1, it follows that

$$
\begin{aligned}
\|y(\mu, \lambda)-\bar{y}\|_{Y} & \leq C\left\|u(\mu, \lambda)+\lambda_{1}-\bar{u}-\bar{\lambda}_{1}\right\|_{L^{p}(\Omega)} \\
& \leq C\left(\|u(\mu, \lambda)-\bar{u}\|_{L^{p}(\Omega)}+\left\|\lambda_{1}-\bar{\lambda}_{1}\right\|_{L^{p}(\Omega)}\right) \\
& \leq C\left(\|u(\mu, \lambda)-\bar{u}\|_{L^{p}(\Omega)}+\|\lambda-\bar{\lambda}\|_{\Lambda}\right) .
\end{aligned}
$$

Combining this with (39), we can assert that there exist positive constants $C_{1}, C_{2}$ satisfying

$$
\|y(\mu, \lambda)-\bar{y}\|_{Y}+\|u(\mu, \lambda)-\bar{u}\|_{L^{p}(\Omega)} \leq C_{1}\|\mu-\bar{\mu}\|_{M}^{\alpha / p}+C_{2}\|\lambda-\bar{\lambda}\|_{\Lambda}^{1 / p} .
$$

This implies that $\|z(\mu, \lambda)-z(\bar{\mu}, \bar{\lambda})\|_{Z} \rightarrow 0$ as $(\mu, \lambda) \rightarrow(\bar{\mu}, \bar{\lambda})$. We obtain the desired property. It remains to show that the solution mapping $z(\cdot, \cdot)$ is Hölder continuous in a neighborhood of $(\bar{\mu}, \bar{\lambda})$. From (40) we can choose neighborhoods $M_{1} \subset M_{0}$ of $\bar{\mu}$ and $\Lambda_{1} \subset \Lambda_{0}$ of $\bar{\lambda}$ such that $z(\mu, \lambda) \in \operatorname{int} B_{Z}\left(\bar{z}, \epsilon_{2}\right)$, for all $\mu \in M_{1}, \lambda \in \Lambda_{1}$. Combining this with (17) yields

$$
N(z(\mu, \lambda) ; K(\lambda))=N\left(z(\mu, \lambda) ; K(\lambda) \cap Z_{0}\right) \quad \forall \mu \in M_{1}, \lambda \in \Lambda_{1} .
$$

From this and (36) we obtain

$$
0 \in F_{z}(z(\mu, \lambda), \mu)+N(z(\mu, \lambda) ; K(\lambda)) \quad \forall \mu \in M_{1}, \lambda \in \Lambda_{1} .
$$

This is equivalent to

$$
\left\langle F_{z}(z(\mu, \lambda), \mu), z-z(\mu, \lambda)\right\rangle \geq 0 \forall z \in K(\lambda), \mu \in M_{1}, \lambda \in \Lambda_{1} .
$$

Consequently, for each $(\mu, \lambda) \in M_{1} \times \Lambda_{1}, z(\mu, \lambda)$ is the unique solution of $P(\mu, \lambda)$. Let $\left(\mu^{1}, \lambda^{1}\right),\left(\mu^{2}, \lambda^{2}\right) \in M_{1} \times \Lambda_{1}$. Putting $\lambda=\lambda^{1}$ and $\beta=\lambda^{2}$ in (12), we can find an element $\zeta_{2} \in K\left(\lambda^{2}\right)$ such that

$$
\left\|z\left(\mu^{1}, \lambda^{1}\right)-\zeta_{2}\right\|_{Z} \leq k\left\|\lambda^{1}-\lambda^{2}\right\|_{\Lambda} .
$$

Also, replacing $\lambda=\lambda^{2}$ and $\beta=\lambda^{1}$ in (12), we can find an element $\zeta_{1} \in K\left(\lambda^{1}\right)$ such that

$$
\left\|z\left(\mu^{2}, \lambda^{2}\right)-\zeta_{1}\right\|_{Z} \leq k\left\|\lambda^{1}-\lambda^{2}\right\|_{\Lambda}
$$

Besides, we have

$$
\left\langle F_{z}\left(z\left(\mu^{1}, \lambda^{1}\right), \mu^{1}\right), \zeta_{1}-z\left(\mu^{1}, \lambda^{1}\right)\right\rangle \geq 0 \quad \text { and } \quad\left\langle F_{z}\left(z\left(\mu^{2}, \lambda^{2}\right), \mu^{2}\right), \zeta_{2}-z\left(\mu^{2}, \lambda^{2}\right)\right\rangle \geq 0 .
$$


Combining these with the strong monotonicity of $F_{z}\left(\cdot, \mu^{1}\right)$ (see Lemma 3.1), we have

$$
\begin{aligned}
& \rho\left\|u\left(\mu^{1}, \lambda^{1}\right)-u\left(\mu^{2}, \lambda^{2}\right)\right\|_{L^{p}}^{p} \\
\leq & \left\langle F_{z}\left(z\left(\mu^{1}, \lambda^{1}\right), \mu^{1}\right)-F_{z}\left(z\left(\mu^{2}, \lambda^{2}\right), \mu^{1}\right), z\left(\mu^{1}, \lambda^{1}\right)-z\left(\mu^{2}, \lambda^{2}\right)\right\rangle \\
\leq & \left\langle F_{z}\left(z\left(\mu^{1}, \lambda^{1}\right), \mu^{1}\right)-F_{z}\left(z\left(\mu^{2}, \lambda^{2}\right), \mu^{1}\right), z\left(\mu^{1}, \lambda^{1}\right)-z\left(\mu^{2}, \lambda^{2}\right)\right\rangle \\
& +\left\langle F_{z}\left(z\left(\mu^{1}, \lambda^{1}\right), \mu^{1}\right), \zeta_{1}-z\left(\mu^{1}, \lambda^{1}\right)\right\rangle+\left\langle F_{z}\left(z\left(\mu^{2}, \lambda^{2}\right), \mu^{2}\right), \zeta_{2}-z\left(\mu^{2}, \lambda^{2}\right)\right\rangle \\
= & \left\langle F_{z}\left(z\left(\mu^{1}, \lambda^{1}\right), \mu^{1}\right), \zeta_{1}-z\left(\mu^{2}, \lambda^{2}\right)\right\rangle+\left\langle F_{z}\left(z\left(\mu^{2}, \lambda^{2}\right), \mu^{2}\right), \zeta_{2}-z\left(\mu^{1}, \lambda^{1}\right)\right\rangle \\
& +\left\langle F_{z}\left(z\left(\mu^{2}, \lambda^{2}\right), \mu^{2}\right)-F_{z}\left(z\left(\mu^{2}, \lambda^{2}\right), \mu^{1}\right), z\left(\mu^{1}, \lambda^{1}\right)-z\left(\mu^{2}, \lambda^{2}\right)\right\rangle \\
\leq & 2 l k\left\|\lambda^{1}-\lambda^{2}\right\|_{\Lambda}+2 \epsilon_{2}\left\|F_{z}\left(z\left(\mu^{2}, \lambda^{2}\right), \mu^{2}\right)-F_{z}\left(z\left(\mu^{2}, \lambda^{2}\right), \mu^{1}\right)\right\|_{Z^{*}} \\
\leq & 2 l k\left\|\lambda^{1}-\lambda^{2}\right\|_{\Lambda}+2 \epsilon_{2} l_{0}\left\|\mu^{1}-\mu^{2}\right\|_{M}^{\alpha} .
\end{aligned}
$$

Here we used the fact that

$$
\left\|z\left(\mu^{1}, \lambda^{1}\right)-z\left(\mu^{2}, \lambda^{2}\right)\right\|_{Z} \leq\left\|z\left(\mu^{1}, \lambda^{1}\right)-\bar{z}\right\|_{Z}+\left\|\bar{z}-z\left(\mu^{2}, \lambda^{2}\right)\right\|_{Z} \leq 2 \epsilon_{2} .
$$

Using the inequality $(a+b)^{s} \leq\left(a^{s}+b^{s}\right)$, where $a, b \geq 0$ and $0<s \leq 1$ (see [12, Inequality 2.12 .2$, p.32]), it follows from (41) that

$$
\begin{aligned}
& \left\|u\left(\mu^{1}, \lambda^{1}\right)-u\left(\mu^{2}, \lambda^{2}\right)\right\|_{L^{p}(\Omega)} \\
\leq & \left(2 \epsilon_{2} l_{0} \rho^{-1}\right)^{1 / p}\left\|\mu^{1}-\mu^{2}\right\|_{M}^{\alpha / p}+\left(2 l k \rho^{-1}\right)^{1 / p}\left\|\lambda^{1}-\lambda^{2}\right\|_{\Lambda}^{1 / p} .
\end{aligned}
$$

By Lemma 2.1, we obtain

$$
\begin{aligned}
& \left\|y\left(\mu^{1}, \lambda^{1}\right)-y\left(\mu^{2}, \lambda^{2}\right)\right\|_{Y} \\
\leq & C\left\|u\left(\mu^{1}, \lambda^{1}\right)-u\left(\mu^{2}, \lambda^{2}\right)+\left(\lambda_{1}^{1}-\lambda_{1}^{2}\right)\right\|_{L^{p}(\Omega)} \\
\leq & C\left(\left\|u\left(\mu^{1}, \lambda^{1}\right)-u\left(\mu^{2}, \lambda^{2}\right)\right\|_{L^{p}(\Omega)}+\left\|\lambda^{1}-\lambda^{2}\right\|_{\Lambda}\right) \\
\leq & C\left(2 \epsilon_{2} l_{0} \rho^{-1}\right)^{1 / p}\left\|\mu^{1}-\mu^{2}\right\|_{M}^{\alpha / p}+C\left(\left(2 l k \rho^{-1}\right)^{1 / p}+\left\|\lambda^{1}-\lambda^{2}\right\|_{\Lambda}^{1-1 / p}\right)\left\|\lambda^{1}-\lambda^{2}\right\|_{\Lambda}^{1 / p} \\
\leq & C\left(2 \epsilon_{2} l_{0} \rho^{-1}\right)^{1 / p}\left\|\mu^{1}-\mu^{2}\right\|_{M}^{\alpha / p}+C\left(\left(2 l k \rho^{-1}\right)^{1 / p}+\left(2 r_{0}\right)^{1-1 / p}\right)\left\|\lambda^{1}-\lambda^{2}\right\|_{\Lambda}^{1 / p} .
\end{aligned}
$$

Combining this with (42) yields

$$
\begin{aligned}
& \left\|y\left(\mu^{1}, \lambda^{1}\right)-y\left(\mu^{2}, \lambda^{2}\right)\right\|_{Y}+\left\|u\left(\mu^{1}, \lambda^{1}\right)-u\left(\mu^{2}, \lambda^{2}\right)\right\|_{L^{p}(\Omega)} \\
\leq & l_{1}\left\|\mu^{1}-\mu^{2}\right\|_{M}^{\alpha / p}+l_{2}\left\|\lambda^{1}-\lambda^{2}\right\|_{\Lambda}^{1 / p},
\end{aligned}
$$

where $l_{1}:=(1+C)\left(2 \epsilon_{2} c \rho^{-1}\right)^{1 / p}$ and $l_{2}:=(1+C)\left(2 l k \rho^{-1}\right)^{1 / p}+C\left(2 r_{0}\right)^{1-1 / p}$. The proof of Theorem 1.1 is complete. 


\section{SOME EXAMPLES}

In this section we will give some examples which illustrate Theorem 1.1.

Example 4.1. Suppose that $k=2, p=2, N \in\{2,3\}, \epsilon=\epsilon_{0}>0$ and $\delta=-1$ a.e. in $\Omega$. We consider the problem $P_{1}(\mu, \lambda)$ of finding $u \in L^{2}(\Omega)$ and $y \in Y$ which minimize the cost function

$$
F(y, u, \mu)=\frac{1}{2}\left\|y-y_{d}\right\|_{L^{2}(\Omega)}^{2}+\frac{\gamma}{2}\left\|u-u_{d}\right\|_{L^{2}(\Omega)}^{2}-\int_{\Omega} y \mu_{1} d x-\int_{\Omega} u \mu_{2} d x
$$

with the state equation

$$
\begin{cases}A y=u+\lambda_{1} & \text { in } \Omega \\ y=0 & \text { on } \partial \Omega\end{cases}
$$

and pointwise constraints

$$
\begin{cases}u \geq \lambda_{2} & \text { in } \Omega \\ \epsilon_{0} u+y \geq \lambda_{3} & \text { in } \Omega,\end{cases}
$$

where $\Omega$ is a bounded domain in $R^{N}$ with the Lipschitz boundary $\partial \Omega, y_{d}, u_{d} \in$ $L^{2}(\Omega), \mu=\left(\mu_{1}, \mu_{2}\right) \in M, \lambda=\left(\lambda_{1}, \lambda_{2}, \lambda_{3}\right) \in \Lambda$ with $M=L^{\infty}(\Omega)^{2}, \Lambda=L^{2}(\Omega) \times$ $L^{\infty}(\Omega) \times L^{\infty}(\Omega)$ and $A$ is a strongly elliptic operator.

For $(\bar{\mu}, \bar{\lambda})=(0,0)$, by [2, Lemma 2.4$], P_{1}(0,0)$ has a unique solution.

Then all conditions of Theorem 1.1 are satisfied. Moreover, there exist positive constants $r_{j}, k_{j}$ with $j=1,2$ such that for all $\left(\mu^{i}, \lambda^{i}\right) \in B_{M}\left(0, r_{1}\right) \times B_{\Lambda}\left(0, r_{2}\right)$ with $i=1,2$, one has

$$
\begin{aligned}
& \left\|y\left(\mu^{1}, \lambda^{1}\right)-y\left(\mu^{2}, \lambda^{2}\right)\right\|_{Y}+\left\|u\left(\mu^{1}, \lambda^{1}\right)-u\left(\mu^{2}, \lambda^{2}\right)\right\|_{L^{2}(\Omega)} \\
\leq & k_{1}\left\|\mu^{1}-\mu^{2}\right\|_{M}^{1 / 2}+k_{2}\left\|\lambda^{1}-\lambda^{2}\right\|_{\Lambda}^{1 / 2},
\end{aligned}
$$

where $(y(\mu, \lambda), u(\mu, \lambda))$ is the unique solution of $P_{1}(\mu, \lambda)$.

In fact, in this case we have

$$
F(y, u, \mu)=\int_{\Omega} f(x, y(x), u(x), \mu(x)) d x,
$$

where $f(x, y, u, \mu)=\frac{1}{2}\left|y-y_{d}(x)\right|^{2}+\frac{\gamma}{2}\left|u-u_{d}(x)\right|^{2}-y \mu_{1}-u \mu_{2}$ or

$$
\begin{aligned}
& f_{y}(x, y, u, \mu)=\left(y-y_{d}(x)\right)-\mu_{1}, \\
& f_{u}(x, y, u, \mu)=\gamma\left(u-u_{d}(x)\right)-\mu_{2} .
\end{aligned}
$$


Hence it is easy to see that assumptions $(A 1)$ and $(A 5)$ are satisfied. Obviously, $f(x, y, u, \mu)$ is convex in $(y, u)$ and

$\left|f(x, y, u, \mu)-f\left(x, y, u, \mu^{\prime}\right)\right|=\left|y\left(\mu_{1}-\mu_{1}^{\prime}\right)+u\left(\mu_{2}-\mu_{2}^{\prime}\right)\right| \leq(1+|y|)(1+|u|)\left|\mu-\mu^{\prime}\right|$.

Hence $(A 2)$ is valid. Since

$$
\left(f_{z}\left(x, z_{1}, \mu\right)-f_{z}\left(x, z_{2}, \mu\right)\right)\left(z_{1}-z_{2}\right)=\left(y_{1}-y_{2}\right)^{2}+\gamma\left(u_{1}-u_{2}\right)^{2}
$$

for all $x \in \Omega, z_{i}=\left(y_{i}, u_{i}\right) \in R^{2}$ with $i=1,2$ and $\mu \in R^{2}$, it follows that assumption $(A 3)$ is fulfilled with $\rho=\gamma$. Also, $(A 4)$ is satisfied with

$$
a_{1}=1, a_{2}(|\mu|)=\gamma+\left|\mu_{1}\right|+\left|\mu_{2}\right|, b_{1}=b_{2}=1, H_{1}(|u|)=1+|u|
$$

and

$$
H_{2}(|u|)=1+\left|u-u_{d}(x)\right|, \alpha_{1}=\alpha_{2}=1 .
$$

Thus all conditions of Theorem 1.1 are fulfilled. The conclusion is followed.

It is noted that when $y_{d}=0, u_{d}=0, y_{c}=0$ a.e. in $\Omega$ then condition (6) is not satisfied. Therefore in this case, Theorem 4.2 in [2] is not applicable for Example 4.1.

The next example illustrates Theorem 1.1 for the case where the integrand function $f$ is not a quadratic function.

Example 4.2. Let $k=4, p=2, N \in\{2,3\}$ and $\epsilon(x)=\epsilon_{0}>0, \delta(x)=\bar{\delta} \phi(x)$ a.e. in $\Omega$. Here function $\phi \in L^{\infty}(\Omega)$ and $\bar{\delta} \in R$ are given. We consider problem $P_{2}(\lambda, \mu)$ of finding $u \in L^{2}(\Omega)$ and $y \in Y$ which minimize the cost function

$$
F(u, \mu)=\int_{\Omega} f(y(x), u(x), \mu(x)) d x
$$

with the state equation

$$
\begin{cases}-\Delta y+y=u+\lambda_{1} & \text { in } \Omega \\ y=0 & \text { on } \partial \Omega\end{cases}
$$

and constraints

$$
\begin{cases}u(x) \geq \lambda_{2}(x) & \text { a. e. in } \Omega \\ \epsilon_{0} u(x) \geq \bar{\delta} \phi(x) y(x)+\lambda_{3}(x) & \text { a. e. in } \Omega\end{cases}
$$

where $\mu=\left(\mu_{1}, \mu_{2}, \mu_{3}, \mu_{4}\right) \in M=L^{\infty}(\Omega)^{4},\left(\lambda_{1}, \lambda_{2}, \lambda_{3}\right) \in \Lambda=L^{2}(\Omega) \times L^{\infty}(\Omega) \times$ $L^{\infty}(\Omega)$ and function

$$
f(y, u, \mu)=\frac{1}{2}\left(y-\mu_{1}\right)^{2}+\frac{\gamma}{2}\left(u-\mu_{2}\right)^{2}+\frac{1}{2}\left(y-\mu_{3} u\right)^{2}+\mu_{4} y^{3} .
$$


Here $\gamma$ is a positive constant.

Easily, we see that $P_{2}(0,0)$ has a unique optimal solution $(\bar{y}, \bar{u})=(0,0)$ corresponding to $(\bar{\mu}, \bar{\lambda})=(0,0)$. We shall show that for $\bar{\delta}$ small enough, there exist positive numbers $r_{1}, r_{2}$ such that for each $(\mu, \lambda) \in B_{M}\left(0, r_{1}\right) \times B_{\Lambda}\left(0, r_{2}\right), P_{2}(\mu, \lambda)$ satisfies all conditions of Theorem 1.1. Moreover, there exist positive constants $k_{j}$ with $j=1,2$ such that for all $\left(\mu^{i}, \lambda^{i}\right) \in B_{M}\left(0, r_{1}\right) \times B_{\Lambda}\left(0, r_{2}\right)$ with $i=1$, 2 , one has

$$
\begin{aligned}
& \left\|y\left(\mu^{1}, \lambda^{1}\right)-y\left(\mu^{2}, \lambda^{2}\right)\right\|_{Y}+\left\|u\left(\mu^{1}, \lambda^{1}\right)-u\left(\mu^{2}, \lambda^{2}\right)\right\|_{L^{2}(\Omega)} \\
\leq & k_{1}\left\|\mu^{1}-\mu^{2}\right\|_{M}^{1 / 2}+k_{2}\left\|\lambda^{1}-\lambda^{2}\right\|_{\Lambda}^{1 / 2},
\end{aligned}
$$

where $(y(\mu, \lambda), u(\mu, \lambda))$ is the unique solution of $P_{2}(\mu, \lambda)$.

In fact, since $\bar{\delta}$ is small enough, $(A 5)$ is valid. Obviously, $(A 1)-(A 2)$ are satisfied. It remains to show that $(A 3)$ and $(A 4)$ are satisfied. We have

$$
\begin{aligned}
& f_{y}(y, u, \mu)=\left(y-\mu_{1}\right)+\left(y-\mu_{3} u\right)+3 \mu_{4} y^{2}, \\
& f_{u}(y, u, \mu)=\gamma\left(u-\mu_{2}\right)-\mu_{3}\left(y-\mu_{3} u\right) .
\end{aligned}
$$

The Hessian matrix of $f$ in $(y, u)$ is given by

$$
H_{f}(y, u)=\left[\begin{array}{cc}
2+6 \mu_{4} y & -\mu_{3} \\
-\mu_{3} & \gamma+\mu_{3}^{2}
\end{array}\right] .
$$

By a detailed computation, we get

$$
f_{y y} f_{u u}-f_{y u}^{2}=2 \gamma+\mu_{3}^{2}+6 \mu_{4} y\left(\gamma+\mu_{3}^{2}\right) \geq \gamma,
$$

and

$$
f_{y y}(y, u, \mu) \geq 2-\frac{\gamma}{\gamma+1}=\frac{\gamma+2}{\gamma+1}
$$

for all $y \in R,|y| \leq \frac{\gamma}{6(\gamma+1)}, u \in R,\left|\mu_{3}\right| \leq 1$ and $\left|\mu_{4}\right| \leq 1$. This implies that for each $\mu=\left(\mu_{1}, \mu_{2}, \mu_{3}, \mu_{4}\right) \in R^{4}$ with $\left|\mu_{3}\right| \leq 1$ and $\left|\mu_{4}\right| \leq 1$, the function $f(\cdot, \cdot, \mu)$ is convex on $\left(-\frac{\gamma}{6(\gamma+1)}, \frac{\gamma}{6(\gamma+1)}\right) \times R$. Moreover, for $z_{i}=\left(y_{i}, u_{i}\right) \in\left(-\frac{\gamma}{6(\gamma+1)}, \frac{\gamma}{6(\gamma+1)}\right) \times R, \mu=$ $\left(\mu_{1}, \mu_{2}, \mu_{3}, \mu_{4}\right) \in R^{4}$ with $\left|\mu_{4}\right| \leq 1$, we obtain

$$
\begin{aligned}
& \left(f_{z}\left(z_{1}, \mu\right)-f_{z}\left(z_{2}, \mu\right)\right)\left(z_{1}-z_{2}\right) \\
= & 2\left(y_{1}-y_{2}\right)^{2}+\left(\gamma+\mu_{3}^{2}\right)\left(u_{1}-u_{2}\right)^{2}+3 \mu_{4}\left(y_{1}^{2}-y_{2}^{2}\right)\left(y_{1}-y_{2}\right)-2 \mu_{3}\left(y_{1}-y_{2}\right)\left(u_{1}-u_{2}\right) \\
= & \left(y_{1}-y_{2}\right)^{2}\left(1+3 \mu_{4}\left(y_{1}+y_{2}\right)\right)+\gamma\left(u_{1}-u_{2}\right)^{2}+\left(y_{1}-y_{2}-\mu_{3}\left(u_{1}-u_{2}\right)\right)^{2} \\
\geq & \gamma\left(u_{1}-u_{2}\right)^{2} .
\end{aligned}
$$

Here we used the fact that

$$
1+3 \mu_{4}\left(y_{1}+y_{2}\right) \geq 1-\frac{6 \gamma}{6(\gamma+1)}=\frac{1}{\gamma+1}>0 .
$$


Hence $(A 3)$ is satisfied. On the other hand we get

$$
f_{y}(y, u, 0)=2 y, \quad f_{u}(y, u, 0)=\gamma u
$$

and for any $y, u \in R, \mu=\left(\mu_{1}, \ldots, \mu_{4}\right), \mu^{\prime}=\left(\mu_{1}^{\prime}, \ldots, \mu_{4}^{\prime}\right) \in R^{4}$

$$
\begin{aligned}
& f_{y}(y, u, \mu)-f_{y}\left(y, u, \mu^{\prime}\right)=-\left(\mu_{1}-\mu_{1}^{\prime}\right)-u\left(\mu_{3}-\mu_{3}^{\prime}\right)+3 y^{2}\left(\mu_{4}-\mu_{4}^{\prime}\right), \\
& f_{u}(y, u, \mu)-f_{u}\left(y, u, \mu^{\prime}\right)=-\gamma\left(\mu_{2}-\mu_{2}^{\prime}\right)+u\left(\mu_{3}-\mu_{3}^{\prime}\right)\left(\mu_{3}+\mu_{3}^{\prime}\right)-y\left(\mu_{3}-\mu_{3}^{\prime}\right) .
\end{aligned}
$$

Hence $(A 4)$ is valid. Thus all assumptions of Theorem 1.1 are fulfilled for $P_{2}(\mu, \lambda)$.

\section{REFERENCES}

1. R. A. Adams, Sobolev Spaces, Academic Press, New York, 1975.

2. W. Alt, R. Griesse, N. Metla and A. Rösch, Lipschitz stability for elliptic optimal control problems with mixed control-state constraints, Optimization, 59(6) 2010, 833-849.

3. J. M. Borwein and Q. J. Zhu, Techniques of Variational Analysis, Springer, Berlin, Heidelberg and New York, 2005.

4. H. Brezis, Functional Analysis, Sobolev Spaces and Partial Differential Equations, Springer, 2010.

5. E. Casas, J. C. D. L. Reyes and F. Tröltzsch, Sufficient second-order optimality conditions for semilinear control problems with pointwise state constraints, SIAM J. Optim., 19 (2008), 616-643.

6. E. Casas and F. Tröltzsch, Recent advances in the analysis of pointwise state-constrained elliptic optimal control problems, ESAIM: Control, Optim. Caculus of Variations, 16 (2010), 581-600.

7. M. Chipot, Elliptic Equations: An Introduction Course, Birkhäuser Verlag AG, BaselBoston-Berlin, 2009.

8. A. Domokos, Solution Sensitivity of Variational Inequalities, J. Math. Anal. Appl., 230 (1999), 382-389.

9. D. Gilbarg and N. S. Trudinger, Elliptic Partial Differential Equation of Second Order, Springer-Verlag Berlin Heidelberg, 2001.

10. R. Griesse, Lipschitz stability of solutions to some state-constrained elliptic optimal control problems, J. Anal. Appl., 25 (2006), 435-455.

11. P. Grisvard, Elliptic Problems in Nonsmooth Domains, Pitman, Boston, 1985.

12. G. Hardy, J. E. Littlewood and G. Pólya, Inequalities, Cambridge, At The University Press, 1934.

13. A. D. Ioffe and V. M. Tihomirov, Theory of Extremal Problems, North-Holand Publishing Company, 1979. 
14. B. T. Kien, Lower semicontinuity of the solution set to a parametric generalized variational inequality in reflexive Banach spaces, Set-Valued Analysis, 16 (2008), 1089-1105.

15. K. Malanowski and F. Tröltzsch, Lipschitz stability of solutions to parametric optimal control for elliptic equations, Control Cybern., 29 (2000), 237-256.

16. C. Meyer, U. Prüfert and F. Tröltzsch, On two numerical method for state-constrained elliptic control problems, Opt. Meth. Software, 22 (2007), 871-889.

17. T. R. Rockafellar and R. J.-B. West, Variational Analysis, Springer-Verlag, 1997.

18. N. D. Yen, Hölder continuity of solutions to a parametric variational inequality, Appl, Math. Optim., 31 (1995), 245-255.

19. E. Zeidler, Nonlinear Functional Analysis and Its Applications II/B: Nonlinear Monotone Operators, Springer-Verlag, Berlin, 1990.

20. E. Zeilder, Nonlinear Functional Analysis and Its Applications III: Variational Methods and Optimization, Springer-Verlag, New York 1985.

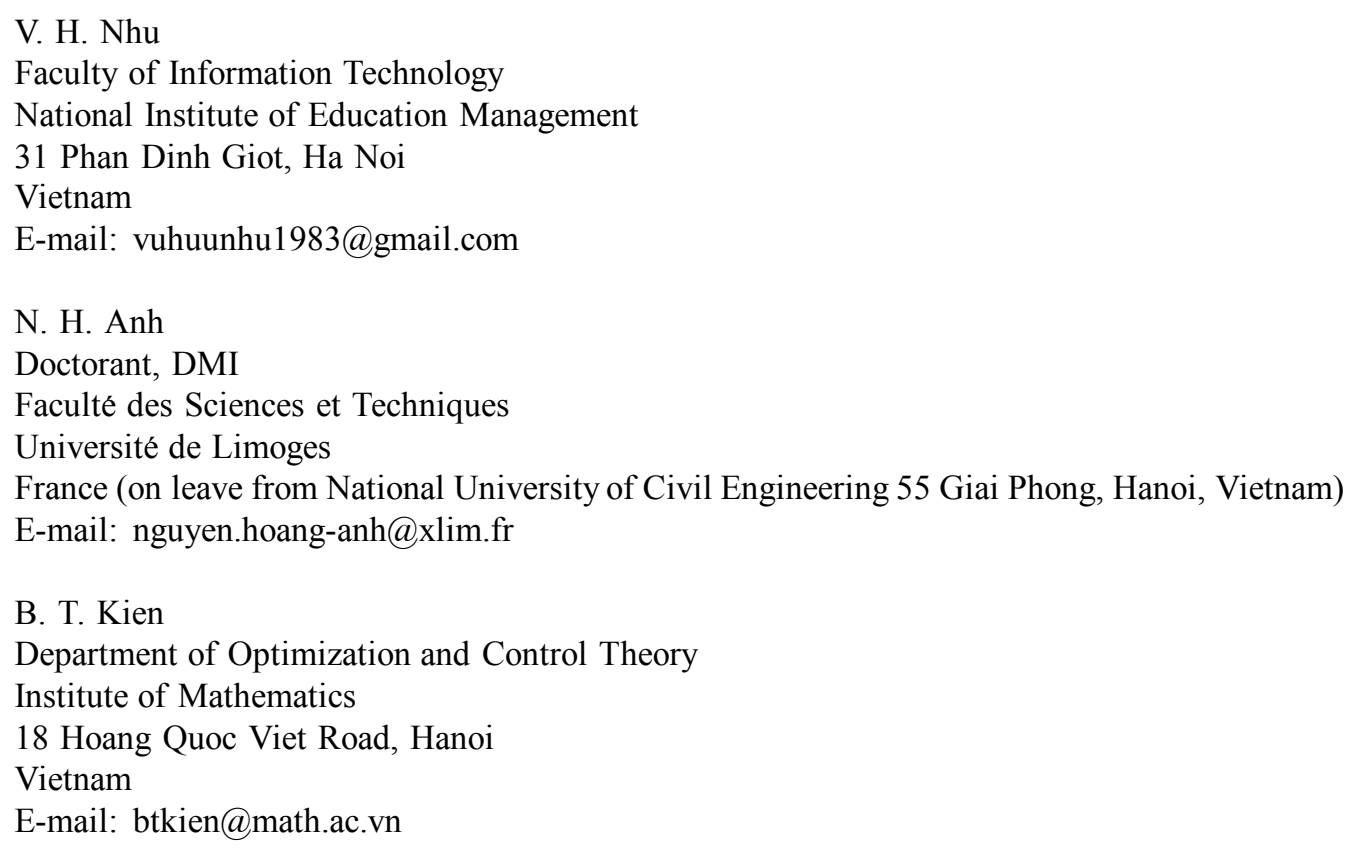

